\title{
Democratizar la bioseguridad en territorios con diversidad biocultural: la apuesta por una alianza de saberes en México
}

\author{
Gabriela Torres-Mazuera ${ }^{\mathrm{a} 1}$ \\ Eric Vides Borrell ${ }^{\mathrm{b}}$ \\ Flor Rivera ${ }^{\mathrm{c}}$ \\ ${ }^{a}$ Centro de Investigación y Estudios Superiores en Antropología Social, México \\ ${ }^{\mathrm{b}}$ El Colegio de la Frontera Sur, México \\ ${ }^{c}$ The Arctic University of Norway, Norway
}

\begin{abstract}
The article focuses on the limitations to citizen participation that the Mexican government has historically imposed in its biosafety regulations and biotechnology policies. It also describes the resistances and creative proposals undertaken by peasants, Indigenous people, scientists and activists concerned about the crop expansion of genetically modified corn and soybean in territories with bio-cultural diversity. Far from assuming an approach that opposes non-academic knowledge to scientific knowledge, our standpoint emphasizes the alliances of knowledge and learnings among multiple actors that make up civil society who advocate for a more democratic and locally anchored biosafety in those territories characterized by their variety and richness in flora, fauna and livelihoods. The article also highlights the contributions that peasants and Indigenous peoples can make, from their territorial knowledge to biosafety democratization, conceived in broader terms than those merely established by law. It also reflects on the challenges and opportunities posed by GMOs citizen biomonitoring, and the controversies that this generates.
\end{abstract}

Keywords: Biosafety regulations, biodiverse territories, citizen biomonitoring, genetically modified organisms, social resistance

\section{Résumé}

L'article se concentre sur les limites à la participation citoyenne que le gouvernement mexicain a historiquement imposées dans ses réglementations en matière de biosécurité et ses politiques en matière de biotechnologie. Il décrit également les résistances et certaines propositions créatives des paysans, des peuples autochtones, des scientifiques et des militants préoccupés par l'expansion du maïs et du soja génétiquement modifiés dans des territoires à diversité bio-culturelle. Loin de supposer une approche qui oppose les connaissances non académiques aux connaissances scientifiques, notre point de vue met l'accent sur les alliances de connaissances et d'apprentissage entre les multiples acteurs qui composent la société civile. Ils préconisent une biosécurité plus démocratique et ancrée localement dans ces territoires caractérisés par leur variété et leur richesse en flore, faune et moyens de subsistance. L'article met également en évidence les contributions que les paysans et les peuples autochtones peuvent apporter, de leurs connaissances territoriales à la démocratisation de la biosécurité. Ces contributions sont bien plus que celles simplement établies par la loi. Nous réfléchissons également aux défis et opportunités posés par la biosurveillance citoyenne des OGM et aux controverses que cela génère.

Mots clés: Réglementations de biosécurité, territoires biodiversifiés, biosurveillance citoyenne, organismes génétiquement modifiés, résistance sociale

\footnotetext{
${ }^{1}$ Gabriela Torres-Mazuera es profesora-investigadora del Centro de Investigación y Estudios Superiores en Antropología Social (CIESAS), México. Email: gtorres-mazuera "at" ciesas.edu.mx. Eric Vides es post-doctorante en El Colegio de la Frontera Sur, Chiapas. Email: erviboro "at" gmail.com. Flor Rivera es estudiante de doctorado en The Arctic University of Norway. Email: flor.r.lopez "at" uit.no. Agradecemos a ConACYT que en el marco del proyecto Ciencia básica 220667, titulado "La privatización de la propiedad social en México" financió parte de la investigación empírica presentada en este texto. También al proyecto LMI-Mobilités, gouvernances et ressources dans le basin méso-américain, financiado por el Institut de Récherche pour le Dévelopement (Francia) y CIESAS-México. Por último, agradecemos a los dos dictaminadores anónimos y a Rodrigo Llanes por sus valiosos comentarios que contribuyeron a mejorar sustancialmente este trabajo.
} 


\section{Resumen}

El siguiente artículo trata sobre las limitaciones al control ciudadano y comunitario impuestas por el gobierno mexicano sobre la bioseguridad y las políticas de biotecnología, así como las resistencias y propuestas creativas emprendidas por campesinos, indígenas, científicos y activistas preocupados por la expansión de los cultivos de soya y maíz genéticamente modificados. Lejos de retomar una perspectiva que opone saberes locales no académicos a conocimiento científico, nuestra perspectiva enfatiza la alianza de saberes y aprendizajes entre múltiples actores que conforman la sociedad civil que abogan por un bio-control más democrático y anclado, a nivel local, en aquellos territorios caracterizados por su variedad y riqueza en flora, fauna y modos de vida. A partir de información etnográfica recabada durante la realización de biomonitoreos en Oaxaca y Campeche entre el 2000 y el 2018, se destacan las contribuciones que campesinos e indígenas pueden hacer, desde su saber territorial, a la democratización de la bioseguridad concebida, esta última, en términos más amplios que los meramente establecidos por ley. Se reflexiona además sobre los retos y oportunidades que suponen los biomonitoreos ciudadanos y las controversias que estos suscitan.

Palabras clave: Bioseguridad, alianza de saberes, territorios biodiversos, biomonitoreos ciudadanos, transgénicos, resistencia social

\section{Introducción}

El siglo XXI mexicano es testigo de dos experiencias de resistencia ciudadana relativamente exitosas contra la presencia y cultivo de organismos genéticamente modificados (OGM). Por organismo genéticamente modificado entendemos un organismo que ha adquirido una combinación genética novedosa por medio de técnicas de biotecnología (ingeniería genética) (LBOGM 2005). La primera experiencia, es la lucha de organizaciones campesinas nacionales y locales, redes de activistas y científicos que desde el 2001 se oponen a la presencia y producción comercial de maíz genéticamente modificado en territorio nacional. Más recientemente, desde el 2012, se desarrolla la batalla de apicultores y campesinos mayas en alianza con activistas, comercializadores y científicos, quienes se oponen a la expansión del cultivo de soya genéticamente modificada en la Península de Yucatán (Campeche, Quintana Roo y Yucatán). Ambas experiencias han revelado la falta de consenso y aceptación social respecto a la introducción de OGMs en el país, así como el poco control que el Estado mexicano tiene sobre el flujo genético de una especie a otra en regiones específicas y, más ampliamente, sobre los cultivos de organismos genéticamente modificados en territorio nacional. En efecto, la contaminación con transgenes ha sido puesta en evidencia, principalmente, por la sociedad civil que se resiste a los transgénicos por medio de múltiples biomonitoreos ciudadanos de OGMs. Lo que se entiende por contaminación de transgénico es un conjunto variado de fenómenos: mezcla accidental de semillas GM con semillas convencionales, la liberación de una variedad de GM sin aprobación, la inclusión de ingredientes GM en comida que se supone es libre es ellos, y casos de flujo de transgenes de un cultivo a otro. En México, el problema de la contaminación se asocia principalmente a la introgresión de constructos transgénicos en poblaciones de maíz criollo en regiones caracterizadas por su diversidad biológica y cultural (Kinchy 2007: 22).

Por biomonitoreo de OGMs nos referirnos al proceso de colecta de muestras de organismos vivos (en estos casos plantas de soya y maíz) para determinar cualitativa o cuantitativamente la presencia de constructos transgénicos o sus expresiones. El concepto de biomonitoreo en sentido amplio, no está asociado específicamente a una técnica de detección, pero para el caso del biomonitoreo de OGMs las técnicas más utilizadas se basan en la detección de proteínas específicas o de ciertas secuencias de ADN. Los biomonitoreos son herramientas con las que cuentan, no solo las instancias del gobierno, sino también los ciudadanos para generar evidencia empírica que en muchos casos contradice o desafía discursos oficiales sobre la inocuidad o supuesta seguridad de proyectos que involucran afectaciones medioambientales. Desde hace tres décadas, los biomonitoreos ciudadanos se han convertido en instrumentos de control y participación ciudadana que logran politizar debates en torno a proyectos tecno-científicos que se presentan como políticamente neutros y científicamente irrefutables (Kimura y Kinchy 2016, 2012; Pearson 2009).

Un aspecto que caracteriza a los biomonitoreos es que estos implican una alianza de saberes entre personas muy diversas, como los son activistas, campesinos, y científicos, con capitales (sociales, económicos y culturales) y trayectorias de vida dispares que logran colaboran en objetivos afines en un momento dado. La alianza de saberes para los biomonitoreos ciudadanos cuestiona, hasta cierto punto, un paradigma tecno- 
científico hegemónico de acuerdo al cual el único conocimiento válido y verdadero para evaluar el riesgo medioambiental, es el producido de manera exclusiva por personas que aplican un conjunto de técnicas y métodos "científicos" y son avalados por instituciones académicas de prestigio nacional e internacional (universidades, revistas, centros de investigación, etc.). Más aun, pone al descubierto el debate contemporáneo entre diferentes comunidades científicas, o epistémicas, en torno a ciertos temas polémicos como son los riesgos que suponen los organismos genéticamente modificados para la salud humana y el medio ambiente (Bonneuil 2006). Este último aspecto es particularmente relevante.

Una de las controversias científicas con más visibilidad en medios de comunicación y la opinión pública en México está encarnada en dos grupos de científicos con visiones contrastadas sobre la bioseguridad y los OGMs. Por un lado, la Academia Mexicana de Ciencias (AMC) con uno de sus principales representantes, Bolívar Zapata quien es un prestigiosos químico bio-tecnólogo favorable al desarrollo de transgénicos. Y, por otra parte, un grupo de científicos, entre los que destacan prestigiosos ecólogos y biólogos moleculares agrupados en la Unión de Científicos Comprometidos con la Sociedad (UCCS) quienes han aportado abundante evidencia empírica sobre contaminación transgénica de maíces nativos en México. Dicha controversia científica se construye sobre una estructura de relaciones de poder asimétricas, en las que ciertos actores (algunos integrantes de la AMC) han tenido mucho más peso en sus decisiones e injerencia en la formulación de leyes (en particular la Ley de Bioseguridad de 2005) y políticas públicas, que otros contestatarios a los OGMs (De Ita 2012; Massieu y San Vicente 2006). La confrontación y diferencia de visiones de ambos grupos en cuanto la labor científica y en específico sobre los OGMs puede verse en el más reciente libro de Bolívar Zapata (2017), Transgénicos, grandes beneficios, ausencia de daños y mitos publicado por Academia Mexicana de Ciencias. Asimismo en la página de la Unión de Científicos Comprometidos con la Sociedad con múltiple información sobre temas variados entre los que se encuentran los OGMs y sus potenciales efectos para la sociedad y el ambiente (https://www.uccs.mx/).

Es en este escenario contencioso que los biomonitoreos de OGMs ciudadanos son relevantes. Como se mostrará en lo que sigue, éstos han permitido revelar el desinterés, la incapacidad y la falta de voluntad política por parte de la Comisión Intersecretarial de Bioseguridad de los Organismos Genéticamente Modificados (CIBIOGEM) dependencia federal encargada de la regulación de los organismos genéticamente modificados para ejercer un verdadero bio-control, entre el 2001 y el 2017, en territorios con diversidad biocultural (Foyer y Bonneuil 2015; Massieu Trigo 2009; Beristein Navarro 2014; Misión de Observación 2017a-d). Asimismo, han permitido establecer alianzas solidarias entre diversos grupos y sectores de la sociedad civil que buscan incidir en las definiciones y prácticas del desarrollo agropecuario a escala local. En un sentido más amplio, los biomonitoreos ciudadanos de OGMs han puesto en cuestión el enfoque de participación ciudadana pasiva implícito en el Protocolo de Cartagena de bioseguridad y en la Ley de Bioseguridad de Organismos Genéticamente Modificados y son una propuesta concreta para democratizar la política de bioseguridad en México.

Este artículo trata sobre las limitaciones al control ciudadano y comunitario impuestas por el gobierno mexicano sobre la bioseguridad en territorios con riqueza ecológica y cultural asociada a las sociedades indígenas y campesinas, es decir en territorios valiosos por su riqueza biocultural. La noción de territorio biocultural es resultado de un debate en el que participan organizaciones indígenas, campesinas y ambientalistas, así como académicos y activistas quienes señalan el aporte indígena y campesino en la preservación del medio ambiente. La asociación entre diversidad ecologica y cultural ha sido de crucial importancia en la elaboración del Convenio Sobre la Diversidad Biológica que es de carácter vinculante para los países que lo firman, entre los que se encuentra México. Entre los aciertos de este Convenio están el haber incorporado la participación de comunidades indígenas como elemento fundamental para lograr la preservación de la biodiversidad (Escobar 1997). En México la noción ha sido retomado por E. Boege quien enfatiza la participación de los pueblos indígenas en la producción y preservación de lo que identifica como "patrimonio biocultural nacional" (Boege Schmidt 2008: 20).

Nuestro punto de vista es el de las resistencias ciudadanas de un conjunto vasto de personas que imaginan la bioseguridad como un asunto que afecta de manera integral su vida, así como a sus comunidades y seres vivos que conforman el entorno natural. Lejos de retomar una perspectiva que opone saberes locales no académicos a conocimiento científico, nuestra perspectiva enfatiza la apuesta por una alianza de saberes y 
aprendizajes entre personas que abogan por un bio-control más democrático y anclado a nivel local. Se destacan las contribuciones que hombres y mujeres campesinos e indígenas pueden hacer, desde su saber territorial, a la bioseguridad concebida en términos más amplios que los meramente establecidos por ley.

Nuestro trabajo se propone además abrir el debate en torno a lo que significaría la democratización de la bioseguridad. Para ello retomamos la reflexión de muy distintos autores que por caminos disímbolos coinciden en señalar la necesidad de generar diálogos de saberes y espacios de encuentro entre personas con perfiles diversos en la discusión de aquellos proyectos socio-tecnológicos con amplio impacto socio-ambiental (Callon et al. 2001; Escobar 1998; Leff 2004). Por diálogo de saberes en este trabajo vamos a comprender "una construcción colectiva de un significado emergente basada en el diálogo entre personas con diferentes experiencias históricas específicas, cosmovisiones y formas de conocimiento, especialmente frente a nuevos retos colectivos en un mundo cambiante" (Martínez-Torres y Rosset 2014: 982, traducción propia). Esta definición desarrollada para explicar la coordinación de objetivos de un movimiento como la Vía Campesina, nos es particularmente útil por referirse a un movimiento social cuyo éxito reside justamente en el intercambio y circulación de conocimientos que se establece entre personas y organizaciones muy diversas.

Este artículo está compuesto por tres apartados. En el primero ofrecemos un panorama de los OGMs en México y las luchas ciudadanas contra la liberación de maíz y soya transgénicos en Oaxaca y Campeche, dos regiones caracterizadas por su diversidad cultural y ecológica. Nuestra intención es proveer al lector de un contexto que permita comprender el debate mexicano en torno a la bioseguridad en la última década. En el segundo apartado consideramos los mecanismos legales e institucionales que regulan los OGMs en México y las significaciones contrastadas de bioseguridad de campesinos mayas y funcionarios que participaron en una consulta indígena sobre soya GM mandatada por la Suprema Corte de Justicia de la Nación (SCJN) en 2015. En un tercer apartado narramos la puesta en marcha de biomonitoreos ciudadanos en Oaxaca para la detección de maíces nativos contaminados por transgenes y para la detección de soya transgénica en Campeche. Finalizamos con una reflexión comparativa de las dos experiencias en relación a los retos que conlleva la alianza de saberes.

Este trabajo se apoya en las diferentes experiencias adquiridas por los tres autores en periodos y regiones variadas. Por un lado, el acompañamiento en la puesta en marcha de biomonitoreos ciudadanos entre el 2003 y el 2005 a nivel nacional y en Oaxaca durante 2005-2010 en colaboración con una asociación civil (Centro de Estudios para el Cambio en el Campo Mexicano A. C.) que lucha contra la expansión del maíz transgénico en México. Por otro lado, la participación e investigación colaborativa en dos colectivos ciudadanos que surgen en la Península de Yucatán entre el 2012 y el 2015 como respuesta a la expansión de la soya GM y la consulta indígena mandatada por la Corte (Ma OGM y Misión de observación de la consulta maya sobre soya transgénica). Estas experiencias han permitido a los autores recorrer regiones del país, dialogar con diferentes personas involucradas de alguna manera en las resistencias sociales al maíz y soya transgénicos y colaborar activamente en la realización de los biomonitoreos ciudadanos.

\section{Organismos genéticamente modificados en México: expansión, regulación y resistencias}

La historia de los OGMs en México inicia en 1988 cuando fueron otorgados los primeros permisos para "liberar" organismos genéticamente modificados con fines experimentales en el norte de México: un tipo de jitomate genéticamente modificado resistente a insectos y algodón Bt producido por la empresa Monsanto (Sandoval Vázquez 2017; Gupta y Falkner 2006). Hasta el 2004 la autoridad encargada de otorgar los permisos era exclusivamente la Secretaría de Agricultura (SAGARPA) por medio de una norma (NOM-056-FITO) que establecía los procedimientos para la liberación experimental de OGMs, pero no regulaba sobre permisos para extensiones a gran escala (más de 10,000 hectáreas) y comercialización de dichos organismos (Gupta y Falkner 2006; Serratos-Hernández 2009). Entre 1988 y 2004 se había otorgado 317 permisos para 26 cultivos experimentales sobre 667,510 hectáreas en 17 estados de la República (Sandoval Vázquez 2017: 11). Durante este periodo, diferentes grupos de interés público y privado expusieron por múltiples vías la necesidad de establecer una mayor regulación de los OGMs. Para los empresarios y funcionarios del gobierno, principalmente del sector agropecuario, la motivación radicaba en promover la expansión comercial de los 
cultivos de organismos genéticamente modificados en territorio nacional; también estaba el interés de científicos biotecnólogos nacionales que buscaban abrir las vías de investigación sobre estos organismos. A la par, pero con una visión enfocada en la protección a la biodiversidad y las poblaciones campesinas e indígenas, estaban las asociaciones ambientalistas y organizaciones campesinas que propugnaban por el principio precautorio y la formalización de evaluaciones de impacto ambiental para la liberación de OGMs. Existía además la preocupación en torno a la falta de regulación en cuanto a la importación de estos organismos, en particular el maíz transgénico proveniente de Estados Unidos (De Ita 2012; González Aguirre 2004 citado en Kinchy 2007: 63; Gupta y Falkner 2006: 35)

Desde 1999 este conjunto de actores pidió y promovió la formulación de una ley para la regulación de OGM, demanda que desembocó, tras seis años de discusión, en la Ley de Bioseguridad de Organismos Genéticamente Modificados (LBOGM) (Chauvet y Gálvez 2005; Massieu Trigo y San Vicente 2006). Esta Ley fue aprobada por la Cámara de diputados en 2005 con poco consenso de las posiciones encontradas y bajo mucha presión por parte de las empresas trasnacionales comercializadoras de semillas transgénicas (Massieu Trigo 2009).

De acuerdo con especialistas, la nueva legislación en bioseguridad se enfocó en dar certeza jurídica a empresarios de la biotecnología, pero fue poco explícita respecto a las restricciones territoriales para establecer zonas libres de transgénicos en centros de origen y diversificación; tampoco fue detallada en definir los mecanismos de vigilancia y control de los OGMs. Desde la perspectiva de los consumidores, la LBOGM es, además, incompleta ya que no incorporó la etiquetación obligatoria de los organismos genéticamente modificados para consumo humano, la cual era una de las exigencias de diversas organizaciones ambientalistas (Massieu Trigo y San Vicente 2006; Massieu Trigo 2009). No obstante, uno de los principales aciertos de la LBOGM fue incorporar a la Secretaría del Medio ambiente (SEMARNAT) como autoridad contraparte de la Secretaría de Agricultura (SAGARPA) en el otorgamiento de permisos, lo cual permitió formalizar la conservación de la biodiversidad en las consideraciones pertinentes para el otorgamiento de permisos para el cultivo de OGMs. Ambas instancias del gobierno deben, a partir de entonces, evaluar cualquier solicitud de permiso para la siembra de organismos genéticamente modificados considerando caso por caso los posibles impactos ambientales que estos organismos pudiesen tener en ecosistemas específicos (Foyer y Bonneuil 2015: 53; Gupta y Falkner 2006: 34). Además, Articulo 108 de la nueva Ley estableció la consulta previa como mecanismo de participación obligatorio cuando la liberación de OGMs se planteara en una región con población indígena.

A partir de la emisión de la nueva legislación en 2005 y hasta el 2017 habían sido otorgados 595 permisos para el cultivo en las distintas fases (experimental, piloto y comercial) de quince OGMs (canola, algodón, alfalfa, soya, maíz, jitomate, calabaza, clavel, chile, frijol, limón, papa, piña, tabaco y trigo) sobre una extensión total aproximada de 5,776,125 hectáreas, sembrada entre el 2005 y 2017, principalmente en los estados del norte del país (Baja California, Chihuahua, Coahuila, Durango, Jalisco, Nayarit, Nuevo León, San Luis Potosí, Sinaloa, Sonora, Tamaulipas) (Sandoval Vázquez 2017: 11). La mayoría de estos permisos de biotecnología han beneficiado a las empresas privadas. Sandoval Vázquez (2007) señala que de los 853 permisos solicitados entre el 2005 y el 2017 para OGM en distintas fases (experimental piloto y comercial), 379 fueron presentados por la empresa Monsanto y 168 por Bayer (Sandoval Vázquez 2007: 11).

\section{La lucha contra el maíz genéticamente modificado en México, 1999-2018}

La resistencia ciudadana contra el maíz transgénico tiene diferentes motivaciones, aunque su fuerza solo se explica si consideramos el sustrato emotivo asociado al maíz nativo como símbolo de identidad nacional en México (Fitting 2011). A continuación, presentamos un breve recuento de los eventos más relevantes que ilustran la lucha contra el maíz transgénico a fin de dar contexto a la siguiente sección sobre biomonitoreos de OGMs ciudadanos. ${ }^{2}$

\footnotetext{
${ }^{2}$ Para una descripción detallada las resistencias sociales al maíz GM en México se pueden consultar los trabajos de los siguientes autores (Fitting 2011; Foyer 2010; Kinchy 2012; De Ita 2012).
} 
Dos hallazgos por parte de científicos y activistas mexicanos y extranjeros activaron la movilización contra el maíz transgénico sobre el cual pesaba una moratoria de facto desde 1998 para su cultivo en fase experimental y comercial. Dicha moratoria de facto fue establecida por el gobierno mexicano, el cual aplicó el principio precautorio dada la importancia socioeconómica, cultural y alimentaria de los maíces nativos, cuyo centro de origen es el territorio mexicano (Chauvet y Gálvez 2005: 67). En 1999 la asociación Greenpeace México reportó el descubrimiento de este grano mezclado con maíz no transgénico, sin ninguna etiquetación, en un contenedor en el puerto de Veracruz (Greenpeace 1999: 22, citado en Massieu Trigo, et al. 2000: 148; Fitting 2011). Dos años después, en 2001, dos investigadores reportaron la presencia de rastros transgénicos en maíz criollo en parcelas campesinas en la Sierra Norte de Oaxaca y publicaron sus hallazgos en la prestigiosa revista Nature (Quist y Chapela 2001). Un estudio subsecuente del Instituto Nacional de Ecología y CONABIO confirmó estos resultados en diferentes localidades de Oaxaca y Puebla (Fitting 2011; Foyer y Bonneuil 2015).

Dada la importancia agro-ecológica, socio-económica y cultural del maíz en México la noticia de contaminación de maíces nativos generó consternación entre la sociedad civil mexicana y las instancias de gobierno encargadas de la bioseguridad y la protección del medio ambiente (De Ita 2004; Kinchy 2012). Asociaciones de la sociedad civil denunciaron la falta de control por parte de la Procuraduría Federal de Protección al Ambiente (órgano administrativo desconcentrado de la Secretaría de Medio Ambiente y Recursos Naturales con autonomía técnica y operativa) por su incapacidad para prevenir la contaminación transgénica, también la inacción de la Comisión Intersecretarial de Bioseguridad de los Organismos Genéticamente Modificados (CIBIOGEM), creada en 1999 con el objetivo de regular la política de bioseguridad en el país. La CIBIOGEM sustituyó al Comité Nacional en Bioseguridad Agrícola (CNBA) encargado de la bioseguridad en México entre 1995 y 1999. En aquellos años, la visión del CNBA estaba dirigida a la prevención y el enfoque de precaución con relación a los OGMs, en particular existía una preocupación entre los científicos integrantes de esta comisión de conservar el patrimonio y recursos genéticos asociados al maíz en México. (SerratosHernández 2009) (De Ita; 2012; Massieu Trigo 2009: 233).

El debate desarrollado en México en torno al maíz GM encarna las visiones contrastadas sobre el desarrollo agrícola y el futuro del mundo rural y campesino. Los grupos y personas en oposición a los cultivos transgénicos tienen entre sus motivaciones los riesgos ambientales y a la salud humana que suponen estos organismos, pero sobre todo hacen una fuerte crítica al giro neoliberal de la política agrícola del gobierno mexicano que desde la firma comercial del Tratado de Libre Comercio de América del Norte (TLCAN) optó por la importación de maíz "'barato y transgénico"'" con repercusiones claramente negativas para los pequeños productores, los modos de vida de campesinos e indígenas y el riesgo que representan a los centros de origen y diversidad de los maíces criollos (De Ita 2012; Kinchy 2007).

Dada una política de ocultamiento por parte del gobierno respecto a la dispersión del maíz transgénico y la ausencia de estudios sobre las posibles repercusiones de la contaminación transgénica en las variedades de maíz, un grupo de veintiún comunidades campesinas de Oaxaca y tres organizaciones ambientalistas (Greenpeace CEMDA y Unión Mexicana de grupos ambientales) presentaron en 2002 una solicitud a la Comisión para la Cooperación Ambiental (CCA) del TLCAN para que esta instancia realizara un estudio independiente sobre los efectos del maíz transgénico (De Ita 2012; Chauvet y Gálvez 2005; Serratos-Hernández 2009). El informe presentado en 2004 tuvo entre sus conclusiones que la principal fuente de transgenes eran las importaciones de maíz proveniente de Estados Uunidos de América; también que el flujo genético existía entre todas las variedades de maíz, los maíces nativos y el teocintle y, se veía favorecido por el intercambio de semillas que realizaban los campesinos. La CCA emitió un conjunto de recomendaciones al gobierno mexicano, las cuales fueron ignoradas, en particular cuando se formuló la Ley de Bioseguridad de Organismos Genéticamente Modificados (De Ita 2012: 58).

La falta de respuesta estatal a las exigencias ciudadanas para la regulación de los OGMs siguiendo el principio precautorio y una política efectiva de bioseguridad ha generado diversas reacciones entre las organizaciones de la sociedad civil que se oponen a los transgénicos. Una de ellas ha sido, en palabras de Ana de Ita (2012) la "defensa social autónoma" (61). Un ejemplo muy ilustrativo de esta respuesta fue la creación en 2002 de la coalición "En Defensa del Maíz." La coalición conformada por organizaciones sociales y ambientalistas en conjunto con grupos campesinos e indígenas de todo el país pugnaba porque los campesinos fuesen tomados en cuenta en las decisiones gubernamentales sobre el uso de biotecnología (Fitting 2011; 
Kinchy 2007, 2012). La coalición de organizaciones que se sumaron a la Red "En defensa del maíz" se propuso como uno de sus objetivos realizar biomonitoreos ciudadanos en comunidades campesinas de todo el país para identificar la posible contaminación de los maíces nativos por flujo transgénico (Kinchy 2010). Entre 2003 y 2005 organizaciones que participan en la Red evaluaron más de 500 parcelas con milpas campesinas en 150 comunidades de once estados de la República (Oaxaca, Veracruz, Puebla, Hidalgo, Chiapas, Estado de México, Chihuahua, San Luis Potosí, Durango, Tlaxcala, Morelos). Los biomonitoreos ciudadanos revelaron contaminación en parcelas de maíces nativos en 18 de las 104 comunidades muestreadas en nueve estados del país (Rivera Lopez 2009).

Uno de los posibles triunfos de la resistencia ciudadana al maíz GM fue la moratoria de facto que el gobierno mexicano mantuvo hasta el 2009, año en que el presidente de ese momento, Felipe Calderón (20062012) decidió decretar su fin y dar "luz verde a las empresas para solicitar permisos de siembra para distintos eventos transgénicos de maíz" (De Ita 2012: 61). En 2012 cuando era inminente la autorización de 79 permisos para la siembra de maíz transgénico en fase comercial a la empresa Monsanto, la batalla política y legal por parte de la ciudadanía y organizaciones contra estos permisos recurrió a la demanda civil de acción colectiva. El argumento legal que presentaron en 2013 un grupo de 53 personas, entre las cuales se encontraban científicos, defensores de los derechos humanos, artistas y 20 organizaciones de productores campesinos, ambientalistas y consumidores, fue la afectación al derecho humano a la diversidad biológica de los maíces nativos o criollos de México que supondría otorgar permisos para la producción comercial de maíz transgénico en México. Las autoridades y empresas demandadas fueron la Secretaría de Agricultura, Ganadería, Desarrollo Rural, Pesca y Alimentación (SAGARPA) y Secretaría del Medio Ambiente y Recursos Naturales (SEMARNAT) y cuatro empresas trasnacionales de biotecnología agrícola. De acuerdo con Adelita San Vicente, una de las demandantes: "la demanda solicita que los tribunales judiciales declaren que los límites y restricciones establecidos en la Ley de Bioseguridad de Organismos Genéticamente Modificados son ineficientes, pues existe evidencia científica de contaminación transgénica de maíces nativos" (San Vicente Tello y Morales Hernández 2015). En 2018 la batalla legal llevaba cinco años de litigio en 17 juzgados federales. En 2019 existe una suspensión definitiva al cultivo del maíz genéticamente modificado en México hasta que se resuelva el más reciente juicio (Narváez Lozano 2018).

En México, una demanda civil de acción colectiva es una demanda interpuesta frente a un juzgado civil a nombre de uno o unos cuantos individuos en representación de un grupo mucho más grande de individuos que han sido perjudicados de la misma manera por la misma conducta de una corporación, un organismo gubernamental u otro demandado. Su fundamento es ser titular de un derecho difuso, individual o colectivo; es decir, derecho que trasciende la esfera individual o que teniendo este carácter, tiene una relación entre varios titulares que los vincula por circunstancias de hecho o de derecho. En México las demandas civiles colectivas son posibles cuando el Artículo 17 constitucional fue reformado en 2010, así como de un conjunto de leyes en 2011 (Código federal de procedimientos civiles, código civil federal, ley general de equilibrio ecológico y protección al medio ambiente, ley federal de protección al consumidor, entre otras)

\section{La resistencia contra la soya genéticamente modificada en Campeche, 2012-2018}

El otro caso que nos interesa describir es el de la resistencia a la soya GM en la Península de Yucatán. Esta resistencia surgió en el año 2012 cuando la SAGARPA otorgó un permiso a Monsanto para el cultivo en fase comercial de soya genéticamente modificada en una superficie de 253,500 hectáreas en los Estados de Campeche, Yucatán, Quintana Roo, San Luis Potosí, Veracruz, Tamaulipas y Chiapas (Colín 2018; TorresMazuera 2018). Este permiso fue dado con el aval de SEMARNAT, instancia que ignoró los tres dictámenes negativos de las dependencias del gobierno que la conforman: el entonces Instituto Nacional de Ecología (INE), la Comisión Nacional para el Conocimiento y Uso de la Biodiversidad (CONABIO) y la Comisión Nacional de Áreas Naturales Protegidas (CONANP). Las tres instituciones dieron dictámenes negativos en los que señalaban las afectaciones ambientales. Por ejemplo, señalaron el riesgo de contaminación del manto freático por glifosato, los daños a la biodiversidad y los riesgos de contaminación por huracanes que implicaría la siembra de soya transgénica en la Península de Yucatán donde el permiso dado abarcaba 60,000 hectáreas de soya transgénica. 
En ese mismo año, apicultores y grupos apícolas de Yucatán y Campeche interpusieron tres demandas de amparo en juzgados de Yucatán y Campeche respectivamente (Colín 2018; Gómez González 2016; TorresMazuera 2018). Los argumentos centrales de los amparos fueron: las afectaciones que tendría la siembra de soya GM a la apicultura y meliponicultura, actividades fundamentales para los hogares campesinos y mayas del municipio. Asimismo, las afectaciones al medio ambiente por el uso excesivo de herbicidas y la deforestación. A la par, la falta de aplicación del principio precautorio y las violaciones al derecho a la consulta previa, libre e informada de los pueblos y comunidades indígenas, en contravención al Artículo $2^{\circ}$ constitucional y al Convenio 169 de la Organización Internacional del Trabajo (OIT). Por último, se alegó que las autoridades responsables no habían tomado en cuenta las opiniones vinculantes del INE, la CONABIO y la CONANP que desaconsejaron el otorgamiento del permiso (Colín 2018).

En 2014 ambos juzgados dictaron sentencias favorables para los integrantes de las comunidades y asociaciones de apicultores mayas. Sin embargo, Monsanto y el Servicio Nacional de Sanidad e Inocuidad y Calidad Agroalimentaria (SENASICA), dependencia de la Secretaría de Agricultura encargada de otorgar los permisos para OGMs agrícolas, y el Ministerio público interpusieron siete recursos de revisión. Estos fueron resueltos por la Suprema Corte de Justicia de la Nación (SCJN) que en 2015 estableció la prohibición de la siembra de soya transgénica en varios municipios de las entidades de Campeche y Yucatán y ordenó realizar una consulta indígena en los municipios donde residían los demandantes a fin de decidir sobre la soya GM (Colín 2018; Torres-Mazuera 2018). Desde las primeras sesiones de la fase de acuerdos previos de dicha consulta, uno de los temas del debate fue la exigencia de las comunidades mayas para participar en el proceso de inspección y vigilancia que el gobierno debía realizar para evitar que se siguiese sembrando soya transgénica en la región. Esta demanda, como se verá en la siguiente sección fue rechazada por las autoridades.En 2018 la siembra de soya GM es ilegal en la Península de Yucatán, ya que además de la prohibición de la SJCN, en 2017 el SENASICA revocó el permiso otorgado en 2012 a Monsanto en todo el país, debido al hallazgo de plantas de soya GM en áreas no autorizadas en el Estado de Campeche (Colín 2018).

Pese a la prohibición, la soya GM se ha seguido cultivando en varios municipios de Campeche. En efecto, en 2016, Greenpeace México y el Equipo Indignación (organización local de promoción y defensa de los derechos humanos) e integrantes de las comunidades mayas de Hopelchén presentaron denuncias ciudadanas ante el SENASICA proporcionando coordenadas de predios donde se estaba sembrando soya GM de manera ilegal. En 2017 campesinos mayas documentaron la presencia de soya GM en 23,000 hectáreas del Estado de Campeche, también en 2018, cuando la totalidad de las parcelas muestreadas por campesinos y apicultores mayas en el municipio de Hopelchén, Campeche, presentaron cultivos de soya GM (Colín 2018, Comunicación personal de integrante del Ma OGM, octubre del 2018).

\section{La regulación de la bioseguridad en territorios bioculturalmente diversos: visiones encontradas de apicultores mayas y funcionarios de gobierno}

De acuerdo a la Ley de Bioseguridad de Organismos Genéticamente Modificados (LBOGM), la bioseguridad es el conjunto de "acciones y medidas de evaluación, monitoreo, control y prevención que se deben asumir en la realización de actividades con organismos genéticamente modificados, con el objeto de prevenir, evitar o reducir los posibles riesgos que dichas actividades pudieran ocasionar a la salud humana o al medio ambiente y la diversidad biológica, incluyendo los aspectos de inocuidad de dichos organismos que se destinen para uso o consumo humano" (Fracción V. LBOGM 2005) (el subrayado es nuestro). Dicha ley establece las atribuciones de instancias gubernamentales de nivel federal que se encargan de realizar estas acciones y medidas.

La LBOGM forma parte de un conjunto de legislaciones nacionales desarrolladas desde los años 1990 para la regulación y comercialización de los OGMs, (Jansen y Roquas 2008). En un análisis crítico sobre el marco internacional que regula la bioseguridad, en particular el Protocolo de Cartagena (de carácter vinculante y firmado por México en 2003) que se ve expresado en dichas legislaciones nacionales, Dominic Glover argumenta que la bioseguridad, en estos instrumentos de regulación, es un concepto muy acotado que solo sirve para establecer la evaluación y manejo de riesgos al medio ambiente y la salud y deja afuera cualquier consideración en torno a sus impactos sociales, económicos o consideraciones de tipo ético (Glover 2003). En 
sus palabras: "El concepto de bioseguridad refleja una forma convencional de pensar sobre el riesgo, en la que los impactos potenciales de las nuevas tecnologías se consideran principalmente una cuestión técnica para ser identificados y evaluados por los científicos", mientras que el "público" es representado como ignorante y no calificado para participar plenamente en el proceso de definición y evaluación de riesgos (Glover 2003: 4). Es así que el concepto de "bioseguridad" implícito en las legislaciones internacionales y en específico en la mexicana, refleja un consenso acerca de lo que es importante para la biotecnología desde un punto de vista regulatorio; es decir, un conjunto de "riesgos" y "beneficios" definidos estrechamente por la "ciencia" y los "científicos (Glover 2003: 14; Jansen y Roquas 2008).

Lo acotado de la definición de bioseguridad fue, sin embargo, debatida durante las sesiones de la consulta indígena sobre soya transgénica por los apicultores y campesinos mayas del municipio de Hopelchén que luchaban contra la expansión de la soya GM en su territorio. Para este grupo de personas la bioseguridad tenía una denotación muy diferente: esta significaba el control que ellos mismos poseían, como colectividad, sobre el lugar donde vivían y sobre el conjunto de elementos naturales (flora, fauna, tierras y agua) que contribuían a su subsistencia.

Antes de presentar los debates que se desarrollaron entre el 2016 y 2017 en torno a la bioseguridad en esta región, vale la pena señalar algunas de sus particularidades agroecológicas que explican lo que estaba en juego en el litigio contra la soya transgénica. El municipio de Hopelchén es parte de una región conformada por el estado de Campeche Yucatán y Quintana Roo que alberga parte del segundo macizo forestal tropical más grande de América, después del Amazonas. Desde los años 2000, la selva y biodiversidad regional se deterioran a pasos agigantados. Entre el 2005 y el 2015, Hopelchén fue uno de los municipios con mayor tasa de deforestación en México (0.51\% anual) (Ellis et al. 2017). La expansión agroindustrial (principalmente de maíz y soya huasteca y transgénica) y la conversión de los terrenos nacionales a propiedad privada son dos de las principales causas de la deforestación y están asociadas a la llegada de productores menonitas provenientes del norte del país (Durango, Zacatecas, Chihuahua y Tamaulipas) (Ellis et al. 2017). A raíz de esta migración, en la actualidad es posible identificar dos grupos socio-culturales bien diferenciados en la región: por un lado, los habitantes maya hablantes residentes en ejidos (algunos de estos pobladores auto-adscritos como mayas); por otro, los menonitas con una población de 9,936 personas que equivale al $12.3 \%$ de los 37,777 pobladores totales del municipio (INEGI 2011).

La diferenciación entre ambos grupos es muy clara en cuanto fenotipo, las formas de vestir, la lengua, la religión y sus actividades económicas y de subsistencia. Para nuestro caso de estudio resaltamos el contraste entre las actividades agropecuarias. Una de las actividades más prósperas de la región es la apicultura, la cual es una actividad practicada principalmente por los mayas. En 2014, la Península de Yucatán producía casi el 40\% de la miel en México, siendo Hopelchén el municipio más productivo de la región. Se calcula que en este municipio 1500 apicultores mayas obtenían sus principales ingresos con la producción de miel en 2014 (Gómez González 2016). La apicultura en la región está asociada a la conservación de la selva: los apiarios normalmente se encuentran localizados en tierras de uso común de los ejidos donde se encuentran los "montes" o áreas forestales con mayor biodiversidad. Los apicultores en la región han sido defensores históricos de las tierras colectivas, así como de su biodiversidad por los beneficios que les traen. No es casual entonces, que fueran grupos de apicultores quienes iniciaran el litigio contra la soya GM en Campeche. Su saber territorial fue expresado desde las primeras discusiones que se dieron entre el 2016 y 2017 durante las reuniones de acuerdos previos de la Consulta indígena ordena por la Suprema Corte. Durante estas sesiones, campesinos y apicultores mayas expresaron su preocupación sobre un conjunto de cambios en el entorno que venían observando desde finales de la década de 1990 y se habían agravado con la llegada de la soya transgénica en el año 2012. Por un lado, la deforestación de grandes áreas de selva que hasta los años 1990 eran propiedad de la nación pero que fueron privatizadas y vendidas a productores menonitas. A la par, veían el avance de la deforestación en los mismos ejidos en parcelas de variable tamaño. También se mencionaba la desaparición de algunas aguadas, en particular la desecación de la laguna Ik, la contaminación del agua potable de las comunidades con herbicidas como el glifosato, la muerte de pavos de monte que se encontraban en canaletas de las nuevas parcelas de cultivo agroindustrial, la muerte de abejas y la caída de productividad de las colmenas, mayores plagas azotando los cultivos tradicionales, y en general, las sequías cada vez más prolongadas en la región y una mayor variabilidad en las lluvias. Todos estos cambios ambientales sobre el territorio en el cual habían residido por 
generaciones eran denunciados como parte del problema de bioseguridad en la región. Un apicultor maya con liderazgo comunitario lo expresó de manera clara cuando, durante una de las reuniones de la consulta, señaló que la "bioseguridad es seguridad para la vida" (Líder de Bolonchén, 26 de octubre del 2016, Misión de observación de la consulta maya sobre soya transgénica, 2016).

Una de las principales batallas que entablaron los integrantes de las 34 comunidades afectadas por la siembra de soya GM en Hopelchén durante dicha consulta fue exigir a las autoridades que, por un lado, garantizaran que la soya GM no sería cultivada en el municipio, en tanto la sentencia de la SCJN estuviera vigente. También pidieron colaborar con el gobierno, desde su conocimiento territorial, a la vigilancia y prevención de la siembra de soya GM. En cuatro acaloradas reuniones con funcionarios de SENASICA, representantes de las comunidades mayas hicieron una propuesta de monitoreo y exigieron información clara y oportuna sobre el trabajo de vigilancia que se venía haciendo y más importante aún, sobre las sanciones a los infractores de la prohibición.

Las sesiones de consulta se convirtieron en foros híbridos similares a los descritos por Callon et al. (2001). Personas con diferentes trayectorias (activismo, gobierno, mercado), visiones del mundo (campesinos, funcionarios de gobierno, académicos, activistas) y formaciones profesionales (biólogos, abogados defensores de derechos humanos, antropólogos, periodistas, entre otros) reunidas en un mismo espacio para discutir, los riesgos de la soya GM. Esta era la primera experiencia de este tipo en la región donde las políticas públicas que afectan a la población maya jamás habían sido debatidas en público. El empuje de apicultores y demás habitantes de las comunidades de Hopelchén, en particular de mujeres mayas preocupadas por el futuro y salud de sus hijos y comunidades para abrir la discusión fue, sin embargo, sistemáticamente rechazado por las autoridades. $^{3}$

Desde la perspectiva de los funcionarios de SENASICA y CIBIOGEM, la bioseguridad era una noción de carácter muy acotado e incompatible con la discusión o el acompañamiento ciudadanos. En particular, resultaba inconcebible la participación ciudadana en los biomonitoreos que debía realizar SENASICA, ya que ésta no estaba definida en ninguna ley. Frente a la complejidad y diversidad de las denuncias asociadas a los cambios producidos por la soya GM y más ampliamente por la agricultura industrial en la zona, la respuesta oficial respuesta era segmentada: la Secretaría de medio ambiente, era la responsable de deforestación, y más precisamente la procuraduría ambiental (PROFEPA); la Comisión Nacional del Agua (CONAGUA) era la responsable del manejo y regulación del agua potable y de riego, así como de los pozos, y SENASICA, (única institución presente en el debate junto con la CIBIOGEM y la Comisión Nacional para el desarrollo de los Pueblos Indígenas), era solo la encargada de la vigilancia de los organizamos genéticamente modificados cuando se trataba de semillas para la producción, ya que cuando se trataba de semilla para el consumo humano, la responsable es la Secretaria de Salud. De éste modo, la aspiración ciudadana a una aproximación integral del territorio y la bioseguridad, expresada por apicultores y campesinos mayas resultaba imposible desde la óptica estatal. Este aspecto era particularmente perturbador, cuando los funcionarios de SENASICA explicaron que durante sus inspecciones en el municipio habían detectado predios con soya GM, la cual había sido "puesta en el mercado para el consumo humano", ya que, de acuerdo al dictamen de la Secretaría de Salud, este grano era inocuo como alimento. Bajo esa argumentación los funcionarios de la Secretaría de Salud habían autorizado la comercialización de la soya transgénica en la región. En la perspectiva de los funcionarios de SENASICA esta medida era correcta y más específicamente era una "medida de bioseguridad" a fin de evitar la propagación del cultivo de este grano en los siguientes ciclos agrícolas (Misión de Observación 2017c). Esta aproximación al problema generó gran descontento entre los apicultores y campesinos mayas, para quienes resultaba fundamental que el gobierno sancionase a los infractores de la sentencia de la Suprema Corte y definiera un programa de reparación de daños para las comunidades mayas afectadas por el cultivo transgénico (Misión de Observación 2017b, 2017c). No solo eso, desde la perspectiva de los mayas apicultores, las autoridades estaban actuando con lentitud, dando tiempo suficiente a los productores soyeros de cosechar la soya en 2017.

Como se desarrolló en otro trabajo donde describimos el devenir de la consulta indígena sobre soya GM (Torres-Mazuera 2018) durante las seis sesiones celebradas en Hopelchén y Tenabo, los funcionarios de

\footnotetext{
${ }^{3}$ Los detalles de cómo se desarrollaron las seis sesiones de Acuerdo previos de la Consulta sobre soya transgénica se pueden consultar en el siguiente vínculo: https://consultaindigenamaya.org/.
} 
CIBIOGEM dejaron ver que en su perspectiva la resistencia de apicultores mayas a la soya GM surgía de la desinformación e ignorancia de estas personas sobre lo que era un transgénico. Durante la fase informativa llevada a cabo en dos comunidades en 2016, los funcionarios de CIBIOGEM acompañados de científicos especialistas en OGM aunque no específicamente de soya GM, se centraron en explicar desde una perspectiva meramente biológica lo que era un organismo transgénico. La estrategia de los dos científicos invitados fue despolitizar la discusión en torno a la soya GM. También omitieron señalar la controversia científica en torno a la posible contaminación de miel por polen transgénico, los riesgos para la salud humana señalados por la Organización Mundial de la Salud en torno al uso del glifosato, los riesgos a la biodiversidad de flora y fauna local señalados en los dictámenes del INE, CONABIO y CONAMP, y las posibles consecuencias sociales y económicas para las comunidades que tendría la expansión del modelo agroindustrial y la soya GM en la región (concentración de tierras, fin del modelo de auto subsistencia campesino, incremento en el uso de plaguicidas y herbicidas, etc.). Destacó el discurso de uno de los "especialistas" que en una comunidad exhortó a los habitantes a "liberase de sus miedos" (Pérez 2016).

En este escenario de contradicción, segmentación, limitación y claro sesgo de la política pública encargada de la bioseguridad en México, los campesinos y apicultores mayas habían empezado algunas iniciativas de organización intercomunitaria para producir ellos mismos la bioseguridad que aspiraban. Esta labor requería de un conocimiento no solo territorial, sino de identificación y registro científico y técnico que sería facilitado por diversas personas trabajando en la región entre las que destacan los biólogos colaborando con los apicultores que formaban parte de un colectivo que trabajaba desde el año 2012 en la lucha contra la soya GM (Gómez González 2016; Torres-Mazuera 2018).

\section{Biomonitoreos de OGMs ciudadanos: retos y oportunidades en la alianza de saberes}

Aunque la colaboración entre campesinos, investigadores y tomadores de decisiones ha sido aconsejada por sectores académicos que reconocen su aporte en la generación de un conocimiento territorial más integral (Tengö, et al. 2014), hasta la fecha esta ha sido poco practicada en México cuando nos referimos a la bioseguridad. Esto a pesar de las opiniones de especialistas en la materia quienes, por ejemplo, señalan que:

Un gran reto para México es el monitoreo y la vigilancia de los OGM en el ambiente. Por la envergadura de la tarea, se deberá recurrir a varios grupos aliados. Por un lado, un monitoreo inclusivo y extenso debe considerar la participación local de grupos interesados e informados. Por otro, se debe instrumentar una red de laboratorios que fortalezca a los que ya está operando el gobierno. (Acevedo Gasman et al. 2009: 348).

Esta labor de monitoreo la han realizado organizaciones y colectivos de la sociedad civil preocupados por la inacción gubernamental frente a la contaminación transgénica del maíz nativo y el cultivo ilegal de la soya GM en la Península de Yucatán.

Las experiencias de biomonitoreo ciudadano de OGMs han sido posible gracias a la alianza de saberes de campesinos, científicos y activistas con diferentes formaciones profesionales. Como veremos en lo que sigue, cada conjunto de actores ha realizado un aporte fundamental desde experiencias y prácticas muy diversas que en un momento dado convergen en un objetivo común. La primera experiencia de biomonitoreo participativo que presentamos sucede en el estado de Oaxaca en 2003, región donde tienen sede varias de las organizaciones de base, campesinas e indígenas que fueron parte de la "Red en Defensa del Maíz". Entre 2003 y 2005 dos organizaciones nacionales, Centro de Estudios para el Cambio en el Campo Mexicano A. C. (CECCAM) y del Centro nacional de ayuda a las Misiones indígenas A. C. (CENAMI) en colaboración con dichas organizaciones locales, realizaron talleres informativos con un enfoque de educación popular en las comunidades de Oaxaca (Valles Centrales, Sierra Sur, Sierra Juárez, Mixteca e Istmo de Tehuantepec) donde se llevaron a cabo los primeros biomonitoreos ciudadanos (Rivera Lopez 2009). En 2005, los campesinos de comunidades en la Sierra Juárez, la Mixteca y los Valles Centrales de Oaxaca, solicitaron al CECCAM y CENAMI, analizar plantas de maíz nativo con deformaciones ya que temían que estas anomalías fuesen consecuencia de la contaminación transgénica. Entre 2005 y 2006, el CECCAM recolectó 301 plantas consideradas como 
anormales en 23 comunidades para ser analizadas. Las plantas fueron colectadas en compañía de autoridades comunitarias y campesinos (en particular aquellas personas de mayor edad y experiencia) quienes identificaron las plantas como "nunca antes vistas" en sus comunidades. El análisis de plantas se realizó con un kit de detección ELISA. Esta técnica, accesible y sencilla, es científicamente valida y presenta niveles de precisión similares a las técnicas de detección basadas en ampliación de ADN (PCR). Los análisis mostraron que una de cada quince plantas deformadas contenían entre una y tres proteínas transgénicas (Rivera Lopez 2009).

A pesar del éxito en términos de cobertura territorial y participación ciudadana de los biomonitoreos realizados por campesinos y activistas, estos fueron ignorados como evidencia empírica de la extensión de flujo transgénico de maíz en México, y fueron descalificados y catalogados como alarmistas por las autoridades gubernamentales (De Ita 2012; Kinchy 2010; Serratos Hernández 2009). No obstante, debido al interés de los campesinos por conocer la situación de sus parcelas y comunidades, los biomonitoreos ciudadanos continuaron entre el 2006 y el 2010, coordinados por CECCAM. Vale la pena una pequeña explicación sobre las posibles razones por la cuales los dichos biomonitoreos no fueron tomados en cuenta por las autoridades. En primer lugar, es necesario subrayar que los biomonitoreos de OGMs no son políticamente neutrales. Como bien lo señala Kinchy (2012) la definición misma de quién monitorea, dónde se recolectan las muestras, qué técnicas se utilizan para hacerlo, cuáles son los métodos de análisis del muestreo, y dónde se publican los resultados son asuntos contenciosos (77). En segunda instancia, es importante tener en mente que el debate en torno a la presencia (o no) transgenes en variedades de maíz criollo ha sido un tema controvertido entre la misma comunidad científica. Bonneuil, Foyer y Wynne (2014) explican con gran detalle las dificultades a las que diferentes equipos de investigación se enfrentaron en la década del 2000 para publicar los resultados de sus trabajos cuando estos reportaban presencia de transgenes en maíces nativos. De la compleja discusión que involucra la aceptación o rechazo de las investigaciones por revistas de prestigio internacional como Nature y Science, retenemos dos conclusiones a las que estos autores llegan. La metodología de mayor validez entre la comunidad científica y gubernamental mexicana para determinar presencia de transgenes es el PCR, una técnica de la biología molecular para amplificar secuencias de ADN que realizan laboratorios especializados en el mundo. Más interesante aún, los resultados con reconocimiento institucional han sido realizados en un mismo laboratorio con sede en Estados Unidos. En cambio, las pruebas ELISA que utiliza un método inmunológico para la detección, fueron consideradas como poco fiables por la comunidad científica mexicana, y en particular por las autoridades encargadas de la bioseguridad, cuando se trataba de demostrar la contaminación transgénica de maíces nativos. La preferencia por un método de análisis sobre otro, tiene consecuencias graves sobre la aspiración de los biomonitoreos ciudadanos ya que conlleva a que solo científicos muy especializados (biólogos moleculares) puedan realizar biomonitoreos que logren resultados con validez necesaria desde la perspectiva de los funcionarios públicos en México. Sin embargo, Bonneuil y colaboradores (2004) contrastan la manera en que en Estados Unidos muestras y análisis con el método ELISA utilizados por organizaciones de la sociedad civil para denunciar contaminación de maíz transgénico no apto para el consumo humano han sido aceptados como válidos y han tenido un impacto político y económico contundente.

Los biomonitoreos ciudadanos realizados en Oaxaca han tenido, sin embargo, otros efectos positivos desde las perspectivas de activistas involucrados. Los biomonitoreos sirvieron como un "pretexto" para difundir información sobre los OGMs en comunidades y afianzar la defensa del territorio y sus recursos de las organizaciones campesinas locales que participaron en el proceso (Kinchy 2012). También fortalecieron la valoración y significado del maíz nativo entre muchos de los campesinos participantes. En varias comunidades, los biomonitoreos, modificaron la práctica agrícola: por ejemplo, en San Pedro Totoltepec y el Limón en el Istmo de Tehuantepec se crearon parcelas comunitarias para sembrar variedades nativas de maíz a fin de generar un intercambio de semillas. También se definieron nuevas estrategias legales, por ejemplo, en la región Mixe se generaron estatutos comunitarios para auto-declararse territorios libres de transgénicos. A partir de entonces, y de acuerdo a un activista zapoteco de la Sierra de Oaxaca, la siembra de maíz nativo en esta región representa, además de un medio de supervivencia, un acto político de resistencia (Aldo González vocero de la Unión de Organizaciones de la Sierra Juárez de Oaxaca de la Sierra Juárez de Oaxaca, Comunicación Personal, Ciudad de México 2005.)

Otra experiencia más reciente es la de los biomonitoreos ciudadanos en el municipio de Hopelchén, Campeche, realizados entre el 2015 y el 2018. En esta región existía trabajo previo de investigación por parte 
de biólogos de una institución pública de docencia e investigación (El Colegio de la Frontera Sur) que realizaban trabajo de campo para determinar la cantidad de polen de soya (convencional o GM) que las abejas trasladaban a sus panales en paisajes soyeros durante el periodo de floración. Esta investigación generó acercamiento con apicultores preocupados por la contaminación de polen de soya GM en la miel, quienes comercializaban su miel en el mercado europeo, muy estricto con las trazas de transgénicos. El primero biomonitoreo colaborativo entre biólogos, apicultores y activistas a fin de identificar el cultivo de soya GM en el municipio de Hopelchén se realizó en el año 2015. Para ello se organizaron tres equipos para colectar hojas de soya y geo-referenciar las muestras tomadas en varios predios del municipio. Los biólogos enseñaron a los apicultores locales el procedimiento de preparación, aplicación e interpretación de la prueba ELISA que se utilizó para la detección de proteínas transgénicas, también el uso de instrumentos de GPS (Global Positioning System).

En la labor del muestreo el saber territorial de los apicultores fue fundamental ya que eran ellos quienes conocían los caminos y brechas poco transitados donde se recolectaron muchas de las muestras. Ellos también estaban alertas de cultivos tempranos de soya (previos al ciclo) que productores locales llevaban a cabo a fin de evadir la prohibición de la siembre de soya GM. Realizar el monitoreo con personas de la región era necesario por las tensiones asociadas a la ilegalidad de la siembra de soya GM. En 2018, por cuarto año consecutivo, un conjunto de personas que participaron activamente en el colectivo Colectivo de comunidades mayas de los Chenes (Hopelchén) realizaron el biomonitoreo. Las brigadas colectaron muestras de soya en parcelas ejidales en todo el municipio. El criterio para tomar o no la muestra fue deliberadamente sesgado ya que su objetivo era detectar mayor cantidad de parcelas de soya GM a fin de denunciar su siembra ilegal. A ese respecto es importante considerar el aprendizaje de los apicultores que a lo largo de tres años de hacer biomonitoreos desarrollaron una mirada "especial" para detectar a simple vista plantas de soya transgénica. De acuerdo a los apicultores mayas que participaron en los biomonitoreos, las plantas de variedades de soya transgénica tienen un tono verde más claro y una talla más baja que las variedades de soya Huasteca. Esta experiencia les permitió acertar con cero margen de error sobre la naturaleza transgénica de las plantas de soya: en 2018 el biomonitoreo dio como resultado un $100 \%$ de muestras positivas a la prueba de detección con kit de ELISA. Los resultados encontrados por los apicultores de Hopelchén tuvieron suficiente peso para movilizar a funcionarios de SENASICA en la búsqueda de las parcelas de soya, quienes incluso pidieron la ubicación de las parcelas, lo cual da cuenta de la credibilidad del monitoreo ciudadano en la región. Esta experiencia deja ver la importancia que tiene el trabajo de muestreo en el éxito de los biomonitoreos de OGMs.

En nuestra reflexión sobre la alianza de saberes para la bioseguridad en México es fundamental reconocer, además, el aporte de los saberes activistas. Las personas que trabajan y colaboran en asociaciones civiles involucradas en la resistencia y lucha contra los OGMs en México y cuentan con experiencia indispensable para que los biomonitoreos ciudadanos sean realizables y tengan una repercusión política. Entre los saberes activistas reconocemos la forma de encuadrar un hallazgo como problemática social, por ejemplo, redefinir la contaminación del maíz GM como un problema de seguridad nacional. Por otro lado, los activistas saben cómo conseguir los fondos para llevar a cabo los biomonitoreos, los cuales son costosos para campesinos por los gastos en la adquisición de los kits de detección y la gasolina para los traslados. De igual o mayor importancia son las redes de apoyo con las que los activistas cuentan, no solo entre organizaciones afines sino sobre todo con medios de comunicación que permiten la divulgación de los resultados de los biomonitoreos (ruedas de prensa, elaboración de infografías, páginas web, etc.).

Las experiencias en Campeche y en Oaxaca revelan la importancia de la participación ciudadana para velar por la bioseguridad en una región específica. Solo los habitantes de una región pueden estar al tanto de los cambios en los ecosistemas, relativos a factores exógenos como cultivos transgénicos. Solo ellos, los directamente afectados, tienen la motivación y legitimidad suficiente para generar mecanismos de vigilancia y control con una cobertura suficiente que garantice, en sentido integral, la bioseguridad en un territorio rural donde las instituciones del estado ya sea que tienen poco alcance o muy poca voluntad para hacerlo. Este último asunto es clave para comprender el fracaso de la bioseguridad en México: la breve, pero intensa, historia de la producción de soya GM en la Hopelchén revela el claro interés por parte de estas instituciones de gobierno para promover el cultivo de OGMs. En este contexto se hace más necesaria que nunca una discusión y acciones concretas donde participen actores de la sociedad civil con diferentes perspectivas en torno a los OGMs. 


\section{Reflexiones finales}

Las experiencias de biomonitoreos ciudadanos que hemos descrito son la prueba del gran potencial que tiene la alianza de saberes para alcanzar una bioseguridad democrática y eficiente en regiones con riqueza biocultural. En esta alianza destaca la participación campesina e indígena que hizo posible llevar a cabo biomonitoreos con una amplia cobertura territorial que muy difícilmente podría realizar el gobierno. Asimismo, el conocimiento de estas personas de su entorno local permitió la identificación de cambios relevantes que pueden apuntar a posibles consecuencias de OGMs, así como la identificación de posibles fuentes y medios de dispersión de la contaminación.

La alianza de saberes para los biomonitoreos de OGMs significa "suma de saberes complementarios" para alcanzar un fin concreto. Los saberes territoriales de campesinos e indígenas se conjugaron exitosamente al conocimiento técnico y científico que aportaron científicos con el objetivo de superar las limitaciones técnicocientíficos impuestas por la LBOGM. Asimismo, el conocimiento activista permitió la divulgación de resultados y la definición de una problemática social en términos de contaminación ilegal transgénica.

La alianza de saberes tiene, sin embargo, sus límites. Aunque ha sido efectiva en generar un tipo de participación activa para velar por la bioseguridad y generar información sobre la contaminación transgénica en territorios con diversidad biológica y cultural, ha sido prácticamente nula en abrir un debate más amplio en torno a la definición misma de bioseguridad. La política de biotecnología en México continúa siendo abordada como un asunto principalmente técnico en el que solo expertos científicos pueden producir la evidencia adecuada para la evaluación necesaria de riesgos, en la toma de decisiones y control de los OGMs. Esta definición legal de bioseguridad que restringe la participación ciudadana, ha sido poco cuestionada tras la emisión de la LBOGM. Entre las razones que explican este desinterés, está el claro apoyo al desarrollo, producción y comercialización de OGM por parte de los gobiernos federales durante tres sexenios (2000-2018). Ante la incapacidad de establecer canales de comunicación y diálogo con el gobierno federal muchas de las organizaciones de la sociedad civil optaron por una defensa autónoma que miraba hacia las comunidades mucho más que hacia el estado. Este panorama ha cambiado con el nuevo gobierno federal (2018-2024) y su presidente que durante su campaña política prometió establecer una prohibición de los OGMs en México. Muchas expectativas han sido generadas en torno a la democratización de la sociedad mexicana, entre las cuales es fundamental incluir la bioseguridad en regiones biodiversas.

\section{Bibliografía}

Acevedo, G.F., E. Huerta, S. Lorenzo and S. Ortiz. 2009. La bioseguridad en México y los organismos genéticamente modificados: cómo enfrentar un nuevo desafío. Capital Natural de México 2: 319-353.

Berinstein Navarro, H. 2014, Democracia participativa, el caso de la apicultura y la introducción de soya genéticamente modificada en Yucatán. Tesis para obtener grado en Maestría en Ciencias en espacialidad e ecología Humana. Mérida: CINVESTAV.

Boege Schmidt, E. 2008. El patrimonio biocultural de los pueblos indígenas de México: hacia la conversación in situ de la biodiversidad y la agrodiversidad de los territorios indígenas. México: Instituto Nacional de Antropología e Historia.

Bonneuil, C. 2006. Cultures épistémiques et engagement des chercheurs dans la controverse OGM. Natures Sciences Société 14(3): 257-268.

Bonneuil, C., J. Foyer and B. Wynne. 2014. Genetic fallout in bio-cultural landscapes: molecular imperialism and the cultural politics of (not) seeing transgenes in Mexico. Social Studies of Science 44(6): 901-929.

Callon, M., P. Lascoumes and Y. Barthe. 2001. Agir dans un monde incertain: essai sur la démocratie technique. Paris: Seuil.

Chauvet, M. and A. Gálvez. 2005. Learning about biosafety in Mexico: between competitiveness and conservation. International Journal of Biotechnology 7(3): 62-71.

Colín, M. 2018. Bio(in)seguridad en México. Permiso de soya transgénica para Monsanto y la siembra ilegal de soya en el estado de Campeche. México: Greenpeace. 
De Ita, A. 2004. Maíz transgénico en México: apagar el fuego con gasolina. In Muñoz Rubio, J. (ed.). Alimentos Transgénicos: ciencia, ambiente y mercado: un debate abierto. México: UNAM-CEIICH/SIGLO XXI.

De Ita, A. 2012. La defensa internacional del maíz contra la contaminación transgénica en su centro de origen. El Cotidiano 173: 157-165.

Ellis, E., J. Montero, I. Gómez, L. Porter-Bolland and P. Ellis. 2017. Private property and Mennonites are major drivers of forest cover loss in central Yucatan Peninsula, Mexico. Land Use Policy 69: 474-484.

Escobar, A. 1998. Whose knowledge, whose nature? Biodiversity, conservation, and the political ecology of social movements. Journal of Political Ecology 5: 53-82.

Fitting, E. 2011. The struggle for maize: campesinos, workers and transgenic corn in the Mexican countryside. Durham: Duke University Press.

Foyer, J. and C. Bonneuil. 2015. La bioseguridad mexicana: una actuación de seriedad. Revista Mexicana de Sociología 77(1): 37-68.

Glover, D. 2003. Public participation in national biotechnology policy and biosafety regulation. IDS Working Paper 198. Brighton: Institute of Development Studies.

González Aguirre, R. 2004. La biotecnología agrícola en México: efectos de la propiedad intelectual y la bioseguridad. México: Universidad Autónoma Metropolitana.

Greenpeace, 1999. El maíz producido genéticamente por Novartis: una amenaza para la salud ambiental, humana y animal. Biodiversidad, Sustento y Culturas 21: 20-24.

Gómez González, I. 2016. A honey-sealed alliance: Mayan beekeepers in the Yucatan Peninsula versus transgenic soybeans in Mexico’s last tropical forest. Journal of Agrarian Change 16(4): 728-736.

Gupta, A. and R. Falkner. 2006. The influence of the Cartagena Protocol on biosafety: comparing Mexico, China and South Africa. Global Environmental Politics 6(4): 23-55.

INEGI. 2011. Anuario de estadísticas por entidad federativa. México: Instituto nacional de estadística, geografía e informática.

Jansen, K. and E. Roquas. 2008. Biosafety regulation and global governance: the problem of absentee expertise in Latin America. In G. Otero (ed.). Food for few: neoliberal globalism and biotechnology in Latin America. Austin: University of Texas Press. Pp. 91-114.

Kimura, A. and A.J. Kinchy. 2016. Citizen science: probing the virtues and contexts of participatory research. Engaging Science, Technology, and Society 2: 331-336.

Kinchy, A. 2007. Genes out of place: science, activism and the politics of biotechnology. PhD Dissertation. Madison: University of Wisconsin-Madison.

Kinchy, A. 2012. Seed, science and struggle: the global politics of transgenic crops. Boston: MIT Press.

Leff, E. 2004. Racionalidad ambiental y diálogo de saberes. Significancia y sentido en la construcción de un futuro sustentable. Polis: Revista Latinoamericana 7(1).

Ley de Bioseguridad de Organismos Genéticamente Modificados. 2005. Diario Oficial de la Federación, [Consultado 15 de noviembre 2018]. http://www.diputados.gob.mx/LeyesBiblio/pdf/LBOGM.pdf.

Martínez-Torres, M. and P.M. Rosset. 2014. Diálogo de saberes in La Vía Campesina: food sovereignty and agroecology. Journal of Peasant Studies 41(6): 979-997.

Massieu Trigo, Y. 2000. Consecuencias de la biotecnología en México: el caso de los cultivos transgénicos. Sociológica 15(44): 133-159.

Massieu Trigo, Y. 2009. Cultivos y alimentos transgénicos en México. El debate, los actores y las fuerzas sociopolíticas. Argumentos 22(59): 217-243.

Massieu Trigo, Y. and A. San Vicente Tello. 2006. El proceso de aprobación de la Ley de bioseguridad: Política a la mexicana e interés nacional. El Cotidiano 2(136): 39-51.

Misión de Observación de la Consulta al Pueblo Maya. 2017. Quinto Reporte. [accessed January 12, 2017]. http://consultaindigenamaya.org/reportes 
Narvaez Lozano, A. 2018. La prolongada batalla por el maíz. Nexos, 1 junio 2018, [accessed December 2018]. https://www.nexos.com.mx/?p=37778.

Pearson T. 2009. On the trail of living modified organisms. Cultural Anthropology 24(4): 712-745.

Pérez U, M. Funcionario pide a mayas "liberarse del miedo" y aceptar la soya transgénica. La Jornada, 12 de julio 2016. [accessed June 16 2020]. https://www.jornada.com.mx/2016/07/12/sociedad/031n2soc.

Quist, D. and I.H. Chapela. 2001. Transgenic DNA introgressed into traditional maize landraces in Oaxaca, Mexico. Nature 414(6863): 541-543.

Rivera Lopez, F. 2009. Análisis de la presencia de proteínas de maíces genéticamente modificados en variedades de maíz nativo en México. Tesis de Licenciatura. Ciudad de Mexico: Universidad Nacional Autonoma de Mexico.

Sandoval Vázquez, D. 2017. Treinta años de transgénicos en México (compendio cartográfico), Centro de Estudios para el Cambio en el Campo Mexicano, Misereor, Brot Fur die Welt. [accessed December 12, 2018]. http://ceccam.org/sites/default/files/30_a\%C3\%B1os_transgenicos.pdf.

San Vicente Tello, A. y J. Morales Hernández. 2015. La demanda colectiva contra el maíz transgénico: ciudanía y soberanía alimentaria. Vía Orgánica. September 30. https://viaorganica.org/la-demanda-colectivacontra-la-siembra-de-maiz-transgenico-ciudadania\%E2\%80\%A8y-soberania-alimentaria/

Serratos-Hernández, J.A. 2009. Bioseguridad y dispersión de maíz transgénico en México. Ciencias 92(092).

Serratos-Hernandez, J.A., J.L. Gómez-Olivares, N. Arreortua-Salindas, E. Buendía-Rodríguez, F. IslasGutiérrez and A. De Ita 2007. Transgenic proteins in maize in the Soil Conservation area of Federal District, Mexico. Frontiers in Ecology and the Environment 5(5): 247-252.

Tengö, M., E.S. Brondizio, T. Elmqvist, P. Malmer and M. Spierenburg. 2014. Connecting diverse knowledge systems for enhanced ecosystem governance: the multiple evidence base approach. Ambio 43(5): 579591.

Torres-Martínez, M.E. and P. Rosset. 2014. Diálogo de saberes in La Vía Campesina: food sovereignty and agroecology. Journal of Peasant Studies 41(6): 979-999.

Torres-Mazuera, G. 2018. Nosotros decimos Má: La lucha contra la soya transgénica y la rearticulación de la identidad Maya en la Península de Yucatán. The Journal of Latin American and Caribbean Anthropology 23(2): 1-19.

Zapata, B. (coord.). 2017. Transgénicos, grandes beneficios, ausencia de daños y mitos. México. Academia Mexicana de Ciencias. 